\title{
Article \\ Ethylenediamine and Pentaethylene Hexamine Modified Bamboo Sawdust by Radiation Grafting and Their Adsorption Behavior for Phosphate
}

\author{
Jifu Du ${ }^{1,2,+}{ }^{\oplus}$, Houhua Xiong ${ }^{1,+}$, Zhen Dong ${ }^{3}$, Xin Yang ${ }^{1}{ }^{\oplus}$, Long Zhao ${ }^{3, *}$ and Jun Yang ${ }^{3, *}$ \\ 1 School of Nuclear Technology and Chemistry \& Biology, Hubei University of Science and Technology, \\ Xianning 437100, China; duzidedu@163.com (J.D.); xionghouhua@hbust.edu.cn (H.X.); \\ sophieyangyifan@163.com (X.Y.) \\ 2 Hubei Key Laboratory of Radiation Chemistry and Functional Materials, Hubei University of Science and \\ Technology, Xianning 437100, China \\ 3 State Key Laboratory of Advanced Electromagnetic Engineering and Technology, School of Electrical and \\ Electronic Engineering, Huazhong University of Science and Technology, Wuhan 430074, China; \\ zhendong@hust.edu.cn \\ * Correspondence: zhaolong@hust.edu.cn (L.Z.); jyang@hust.edu.cn (J.Y.) \\ + Both the authors equally contribute to this work.
}

\section{check for} updates

Citation: Du, J.; Xiong, H.; Dong, Z.; Yang, X.; Zhao, L.; Yang, J. Ethylenediamine and Pentaethylene Hexamine Modified Bamboo Sawdust by Radiation Grafting and Their Adsorption Behavior for Phosphate. Appl. Sci. 2021, 11, 7854. https://doi.org/10.3390/app11177854

Academic Editors: Dorota

Swiatla-Wojcik, Yosuke Katsumura and Radosław A. Wach

Received: 2 August 2021

Accepted: 24 August 2021

Published: 26 August 2021

Publisher's Note: MDPI stays neutra with regard to jurisdictional claims in published maps and institutional affiliations.

Copyright: (C) 2021 by the authors Licensee MDPI, Basel, Switzerland. This article is an open access article distributed under the terms and conditions of the Creative Commons Attribution (CC BY) license (https:// creativecommons.org/licenses/by/ $4.0 /)$.

\begin{abstract}
Phosphate is an important component for the growth of plants and microorganisms; however, excess phosphate causes serious eutrophication in natural waters. New potential low-loss adsorbents from natural biomass for phosphate removal are desired. Bamboo is one of the most abundant renewable cellulose resources; however, the pure bamboo cellulose is poor to adsorb phosphate. To enhance the adsorption capacity, in this work, bamboo sawdust (BS) was chemically modified by two kinds of amines. First, glycidyl methacrylate (GMA) was grafted on BS using radiation induced graft polymerization. Then, the GMA-grafted BS was further modified by a ring-opening reaction with amines, including ethylenediamine (EDA) and pentaethylene hexamine (PEHA). The amine groups were then quaternized to prepare the BS-GMA-EDA-Q and BS-GMAPEHA-Q adsorbents. The adsorbents were characterized by FTIR, SEM, TG, and XPS analysis. The adsorption performances of the adsorbents for phosphate were evaluated through batch experiments. The adsorption by BS-GMA-EDA-Q and BS-GMA-PEHA-Q both well obeyed the pseudo-secondorder kinetic model and the Langmuir isotherm model, indicating that the adsorption process was chemical monomolecular layer adsorption. The maximum adsorption capacities for BS-GMA-EDA-Q and BS-GMA-PEHA-Q calculated by the Langmuir model were 85.25 and $152.21 \mathrm{mg} / \mathrm{g}$, respectively. A total of $1 \mathrm{~mol} / \mathrm{L} \mathrm{HCl}$ was used to elute the saturated adsorbents. A negligible decrease in adsorption capacity was found after five adsorption-desorption cycles.
\end{abstract}

Keywords: bamboo sawdust; radiation induced graft polymerization; phosphate adsorption

\section{Introduction}

As an eco-friendly material, bamboo can reach its full growth rapidly in a few months, which is one of the most abundant renewable cellulose resources [1]. Owning to its nontoxicity, cost-effectiveness, and environmentally friendly process, bamboo is widely used in construction and reinforcing fibers, paper, textiles, board, furniture, flooring tiles, food, transportation, and packaging industries, combustion, and other bioenergy applications. However, the overall processing efficiency is relatively low [2]. In recent years, progress has been made in the preparation of bamboo charcoal [3], pulp and cellulose nanofiber [4], bamboo-plastic composite materials [5], antioxidants and immunostimulants [6], and metal ion sensor [7] from bamboo processing residues. This progress shows good prospects for the development and application of bamboo waste [1]. 
Bamboo is a promising regeneration adsorbent for heavy metal adsorption from an aqueous solution [8]. Thus, bamboo-based bio-adsorbents for removing metal ions in an aqueous solution have been developed [9]. Bamboo charcoal and activated carbon are widely used for contaminated wastewater purification [10]. These reports show bamboo has good adsorption capacity for heavy metal ions. Some efforts have developed an efficient adsorbent by increasing the number of pores [11], or by increasing the number of accessible functional groups [12-14]. However, the selected precursor and synthetic conditions influenced the microstructure of bamboo charcoal and activated carbon, thus affecting target ions' removal efficiency [14]. Additionally, bamboo charcoal and activated carbon show poor selective adsorption. Chemically modified bamboo shows a higher adsorption capacity for heavy metals than for activated carbon [8]. The bamboo surface is rich in hydroxyl groups because the main components of bamboo are cellulose and hemicellulose, which account for about $50 \%$ of the dry matter [9]. Chemical modification of the hydroxyl group can prepare an adsorbent with higher binding capacity for target ions [8]. Functional groups, especially amino groups chemically grafted onto cellulose, can increase their adsorption performance $[15,16]$. Some papers focus on the chemical modification of bamboo cellulose and their adsorption performance to heavy metal ions $[9,17,18]$, dyes [12]s and $\mathrm{CO}_{2}$ [19]. However, few papers have investigated the adsorption of modified bamboo to anions.

The amine groups, which can be protonated or quaternized to form positive groups such as $\mathrm{R}_{-} \mathrm{NH}_{3}{ }^{+}$and $\mathrm{R}-\mathrm{NRH}_{2}{ }^{+}$, are often used for anionic contaminants adsorption through electrostatic interaction [20-22]. $\mathrm{R}-\mathrm{NH}_{2}$ can be protonated at acidic $\mathrm{pH}$ and de-protonated at a high $\mathrm{pH}$, so that the amine group can adsorb anions at an acidic $\mathrm{pH}$. However, the quaternized amine can adsorb anions at a wide $\mathrm{pH}$ range even in alkaline conditions [21]. Adsorption commonly occurred at the interface between the adsorbent and the adsorbate in an aqueous solution. More functional groups onto the adsorbent surface can obtain high adsorption capacity and velocity. Radiation-induced graft polymerization (RIGP) is often used to increase functional groups and the capacity to bond anions. RIGP was widely used to modify cellulose biomass because RIGP generates radicals and thus form grafting, which are relevant for subsequent chemical modification and processing. The high grafting yield of RIGP can be achieved quickly and without heating [23]. The functional groups or the intermediate monomer were introduced onto the polymer's surface, so the adsorbents had a high adsorption velocity [24].

Phosphate is the only phosphorus available for plant uptake and is a key limiting nutrient in aquatic ecosystems [25]. The massive discharge of phosphate from human activities and agricultural and industrial production causes eutrophication of waterbodies. It is widely known that the control of phosphate in waterbodies can prohibit eutrophication [26]. Physical, chemical, and biological technologies have been used to remove phosphate from the wastewater. Most of these technologies are available for the removal of high concentration phosphate [27]. Water eutrophication may occur when phosphate in water is maintained at a very low concentration; thus, to protect against eutrophication, phosphate at lower concentrations should be removed.

In this work, two kinds of amines were grafted onto bamboo sawdust (BS) by RIGP technique and further modified to prepare quaternized BS. The phosphate adsorption performance of the two adsorbents before and after quaternization was investigated.

\section{Materials and Methods}

\subsection{Materials}

Bamboo sawdust (BS) was obtained from Phyllostachys pubescens (Xianning City, Hubei, China). The BS was $\mathrm{NaOH}$ treated for $1 \mathrm{~h}$ at $100{ }^{\circ} \mathrm{C}$. Ethylenediamine (EDA) was obtained from Aladdin Chemical Co., Ltd. (Shanghai, China) Pentaethylene hexamine (PEHA) was obtained from Tokyo Chemical industry Co., Ltd. (Tokyo, Japan). HCl, $\mathrm{NaOH}$, 1-bromohexane, DMF, and $\mathrm{NaH}_{2} \mathrm{PO}_{4}$ were supplied by Macklin reagent Co., Ltd. (Shanghai, China). 


\subsection{Preparation of BS-GMA-EDA-Q and BS-GMA-PHEA-Q Adsorbent}

BS-GMA-EDA-Q and BS-GMA-PHEA-Q adsorbents were prepared by RIGP, and the synthesis process is illustrated in Figure 1.

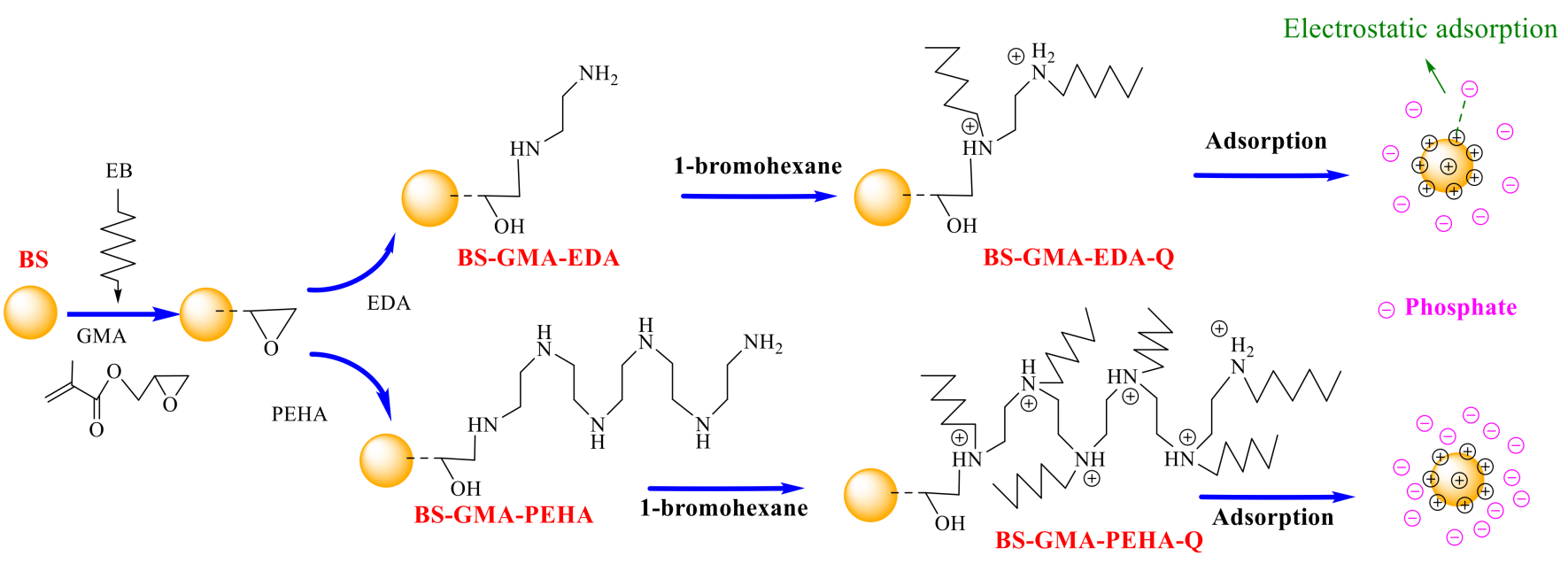

Figure 1. Procedure for the preparation and adsorption mechanism for phosphate of BS-GMA-EDA-Q and BS-GMA-PHEAQ adsorbents.

\subsubsection{EB Radiation Grafting}

An emulsion solution containing 30\% GMA and 3\% Tween 20 was placed into a closed container, and oxygen was removed from the solution by nitrogen flow. Dry BS ( 2 g) was put into PE bags and then vacuum sealed and injected with $30 \mathrm{~mL}$ of GMA emulsion. The bags were placed on an electron beam irradiation device of a car and irradiated by an electron beam under an accelerator (Wasik Associates INC, Dracut, MA, USA) at $1 \mathrm{MeV}$. The electron beam delivered a dose of $10 \mathrm{kGy} /$ pass. Samples were irradiated with a dose of 30-70 kGy. After irradiation, the grafted BS was washed with deionized water, and dried in a vacuum at $50{ }^{\circ} \mathrm{C}$.

The grafting yield (GY) was calculated using Equation (1):

$$
G Y=\left(\frac{W_{g}-W_{0}}{W_{0}}\right) \times 100
$$

where $W_{0}$ and $W_{g}$ were the mass of BS before and after grafting, respectively.

\subsubsection{Preparation of BS-GMA-EDA-Q and BS-GMA-PHEA-Q Adsorbent}

The GMA-grafted bamboo powder (BS-GMA) was further functionalized by amines. BS-GMA $(2.0 \mathrm{~g})$ was immersed into $100 \mathrm{~mL}$ EDA or PEHA with DMF solution at $80^{\circ} \mathrm{C}$ for $24 \mathrm{~h}$. After the ring-opening reaction, the BS-GMA-EDA and BS-GMA-PEHA samples were washed and dried at $50^{\circ} \mathrm{C}$ to a constant weight. The degree of amination (DA) was calculated by Equation (2):

$$
\mathrm{DA}=\frac{\frac{W_{a}-W_{g}}{M w_{a}}}{\frac{W_{g}-W_{0}}{M w_{G M A}}} \times 100
$$

where $W_{g}$ is the weight of BS-GMA and $W_{a}$ is the weight of BS-GMA-EDA and BS-GMAPEHA. $M_{w_{a}}$ is the molecular weight of the amination agent, and the $M_{w}$ of GMA is 142.15 .

The BS-GMA-EDA and BS-GMA-PEHA were quaternized for $24 \mathrm{~h}$ in 1-bromohexane DMF solution at $70^{\circ} \mathrm{C}$. Thus, BS-GMA-EDA-Q and BS-GMA-PHEA-Q were obtained.

\subsection{Characterization}

FTIR spectra were performed in attenuated total reflectance (ATR) mode on a Bruker Tensor 27 (Karlsruhe, Germany) at a wave number range of $4000-400 \mathrm{~cm}^{-1}$. Surface mor- 
phologies were observed by a scanning electron microscope (SEM) (Tescan, Vega3) at a voltage of $10 \mathrm{kV}$. Thermogravimetric (TG) analysis was carried out on a thermogravimetric analyzer (TA instrument mode 600) in a nitrogen atmosphere. X-ray photoelectron spectroscopy (XPS) analyses were conducted using an AXIS-Ultra instrument (Kratos Analytical). The total phosphate concentration $\left(\mathrm{PO}_{4}{ }^{3-}, \mathrm{HPO}_{4}{ }^{2-}\right.$ and $\left.\mathrm{H}_{2} \mathrm{PO}_{4}{ }^{-}\right)$were measured using an Ion chromatograph (MagIC Net 883, Metrohm, Switzerland).

\subsection{Batch Adsorption Experiments}

In the $\mathrm{pH}$ effect and effect of contact time studies, the adsorbents $(0.05 \mathrm{~g})$ were dispersed in phosphate aqueous solution in a beaker containing $50 \mathrm{~mL}$ of $25 \mathrm{mg} / \mathrm{L}$ phosphate solution. The beaker was shaken by a shaker at $120 \mathrm{rpm}$ and $25^{\circ} \mathrm{C}$. Adsorption times varied from 5 to $300 \mathrm{~min}$. In the effect of phosphate concentration study, the phosphate concentration ranged from 25 to $250 \mathrm{mg} / \mathrm{L}$. Different $\mathrm{pHs}$ were adjusted using $0.5 \mathrm{M}$ $\mathrm{HCl}$ or $\mathrm{NaOH}$. The adsorption capacities of phosphate onto the BS-GMA-EDA-Q and BS-GMA-PHEA-Q at different time $\left(Q_{t}\right)$ were calculated by Equation (3):

$$
\mathrm{Q}_{\mathrm{t}}=\frac{\left(\mathrm{C}_{0}-\mathrm{C}_{\mathrm{t}}\right) \times \mathrm{V}}{\mathrm{m}}
$$

where $\mathrm{C}_{0}$ and $\mathrm{C}_{\mathrm{t}}$ were the phosphate concentration before and after adsorption, $\mathrm{V}$ was solution volume and $\mathrm{m}$ was the mass of adsorbent.

\section{Results}

\subsection{Synthesis of the BS-GMA-EDA-Q and BS-GMA-PHEA-Q}

The RIGP technique is mainly dominated by free radical mechanism. The total amounts of free radicals generated in the substrates and the monomer is the main factor affecting the GY. Figure 2a shows the radiation dose effect on the GY. The GY increases with the dose, and it reached a maximum of $239.6 \%$ at $60 \mathrm{kGy}$. However, the GY of GMA decreased when the radiation dose increased above $60 \mathrm{kGy}$. These phenomena can be explained by the decay mechanism of the trapped radicals. The grafting polymerization was mainly controlled by the total amount of free radicals formed in the substrates. The high adsorbed dose will initiate more free radicals, thus introducing a high GY. Nevertheless, the grafting occurred only at the interface of the substrate and solution, so GY no longer increases with the dose increasing, the decomposition of cellulose molecules was also intensified [28]. The multiple influence factors result in the decrease of the GY. In this study, the grafted BS with the GY $239.6 \%$ at $60 \mathrm{kGy}$ were selected for further experiment.

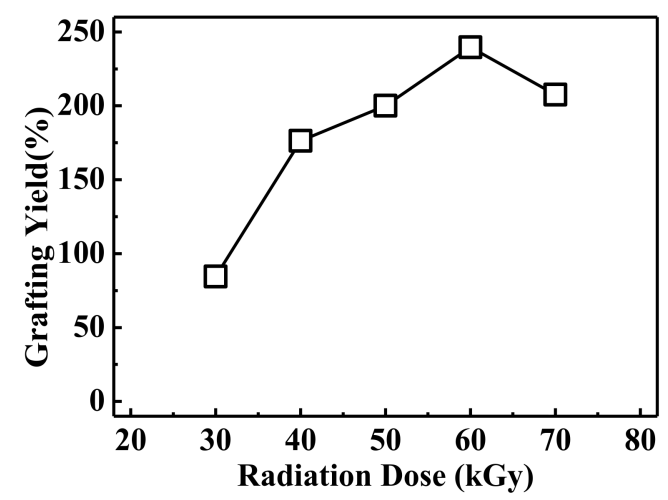

(a)

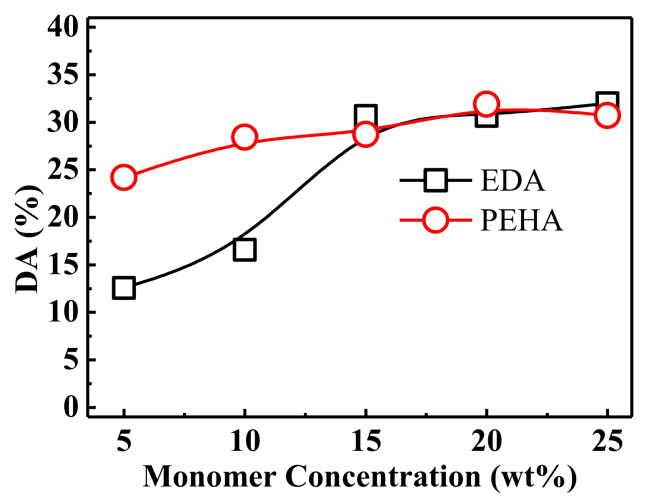

(b)

Figure 2. Effect of radiation dose on GY (a) and effect of monomer concentration on DA (b).

The BS-GMA with the GY $239.3 \%$ is immersed into EDA or PEHA with the DMF solution for the epoxy ring-opening reaction. The effect of the monomer concentration on DA is shown in Figure 2b. DA increased with monomer concentration and reached $31 \%$ 
for both amine monomer at a monomer concentration $20 \mathrm{wt} \%$, implying that BS-GMA is aminated with the same DA by EDA and PEHA. The BS-GMA -EDA and BS-GMA-PEHA at a monomer concentration of $20 \mathrm{wt} \%$ were selected for further experiment.

\subsection{Characterization}

\subsubsection{FTIR Spectra}

Figure 3 shows the FT-IR spectra of BS based adsorbents. The typical absorption bands of cellulose were observed in the curve (a), including 3345, 2920, 1026, and $895 \mathrm{~cm}^{-1}$ due to $\mathrm{O}-\mathrm{H}, \mathrm{C}-\mathrm{H}, \mathrm{C}-\mathrm{O}$ and $\mathrm{C}-\mathrm{O}-\mathrm{C}$ bonding, respectively [29]. The bands at $1724 \mathrm{~cm}^{-1}$ and $905 \mathrm{~cm}^{-1}$ corresponding to the stretching of the carbonyl and epoxy group of GMA confirmed the successful grafting of GMA on BS. After the ring-opening reaction, the bands at $1460 \mathrm{~cm}^{-1}$ are assigned to the N-H bands of EDA and PEHA [22]. The new bands appeared at $1650 \mathrm{~cm}^{-1}$ in curves e and $\mathrm{f}$ are the characteristic bands of quaternary nitrogen, confirming that the tertiary amines of EDA and PEHA were converted into the quaternary ammonium group [30,31].

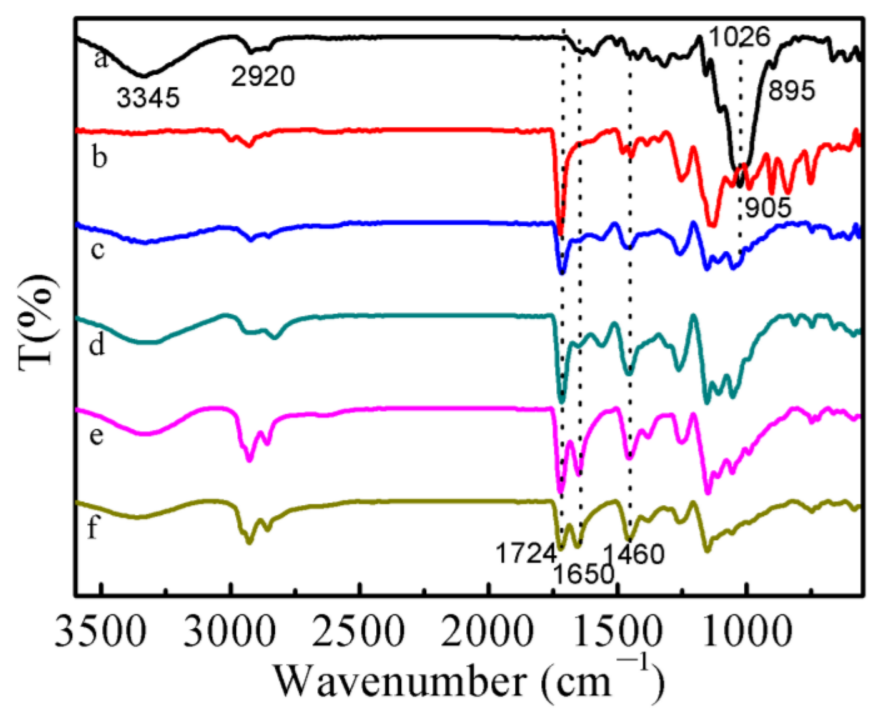

Figure 3. FTIR spectra of original BS (a), BS-GMA (b), BS-GMA-EDA (c), BS-GMA-PEHA (d), BS-GMA-EDA-Q (e), and BS-GMA-PEHA-Q (f).

\subsubsection{SEM Photographs}

SEM photographs of the spectra of BS-based adsorbents are shown in Figure 4 . The surface structure of BS demonstrated ordered fibrils and no other substance attached because lignin was removed by $\mathrm{NaOH}$ treatment. After GMA grafting, the cellulose fiber's regular arrangement on the BS surface was destroyed (Figure 4b). After chemical modification by EDA and PEHA and further quaternization, the BS surface became rough due to the GMA graft polymerization and linkage of multiple amine chain attached to the BS surface (Figure 4c,d). After quaternization, the surface became rougher because of the immobilization of 1-bromohexane. The surface changes of BS before and after grafting, combined with the infrared spectra, confirmed that the chemical modification was successful. 

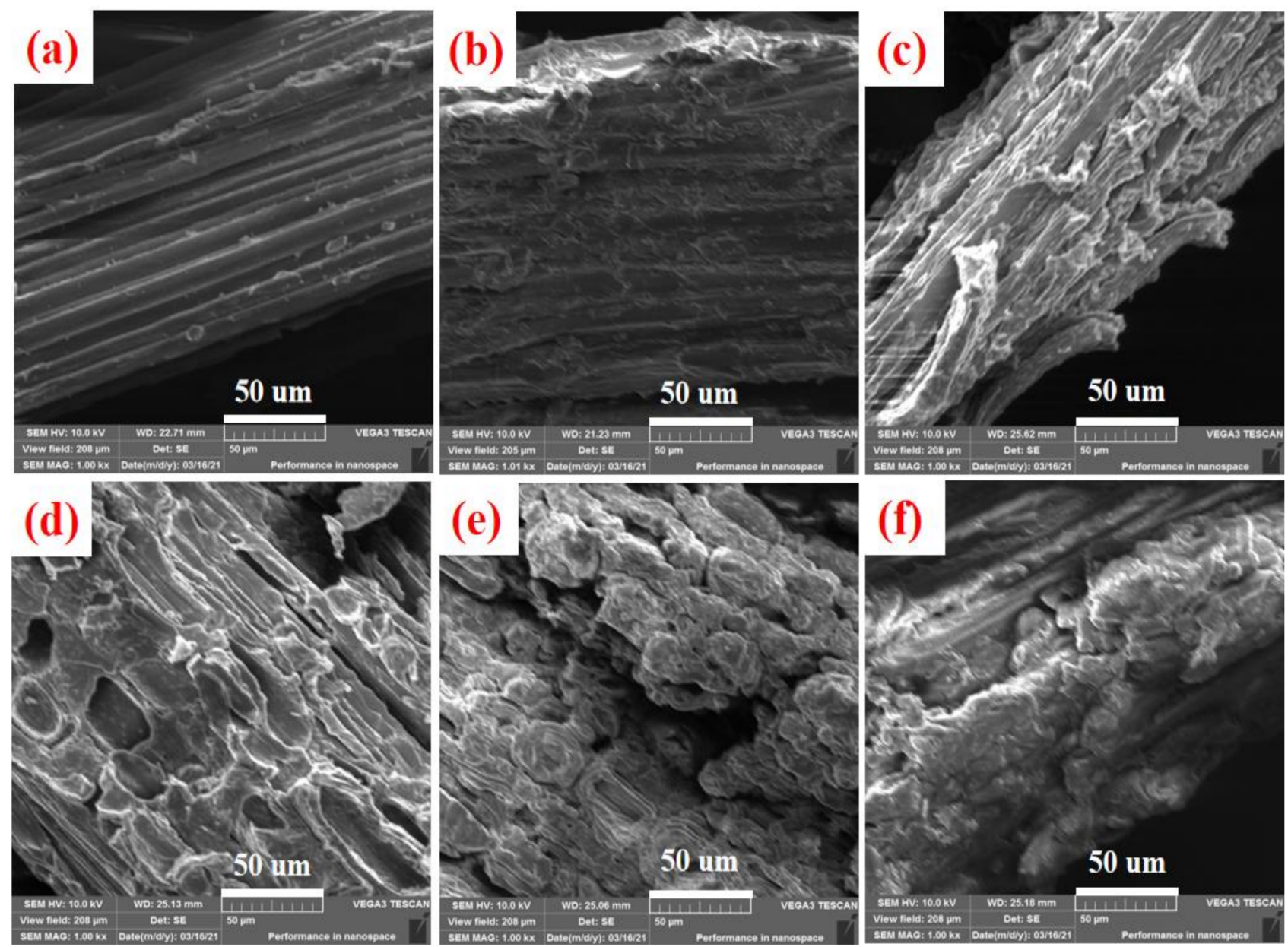

Figure 4. SEM photographs of BS (a), BS-GMA (b), BS-GMA-EDA (c), BS-GMA-PEHA (d), BS-GMA-EDA-Q (e) and BS-GMA-PEHA-Q (f).

\subsubsection{TG Analysis}

Figure 5 shows the TG analysis of BS based adsorbents. BS shows high thermal stability up to $300{ }^{\circ} \mathrm{C}$ and decomposes rapidly above $300^{\circ} \mathrm{C}$, showing a one-step weight loss. The BS-GMA curve (b) shows a gradual weight decrease in the range of $230-400^{\circ} \mathrm{C}$. The weight loss was due to the complex thermal decomposition of the BS and the grafted GMA chains. However, for the aminated BS and quaternized BS, the thermal degradation behavior involved a two-stage process. The principal weight loss occurred between 220 and $450{ }^{\circ} \mathrm{C}$, suggesting that these adsorbents could be used for phosphate adsorption. 


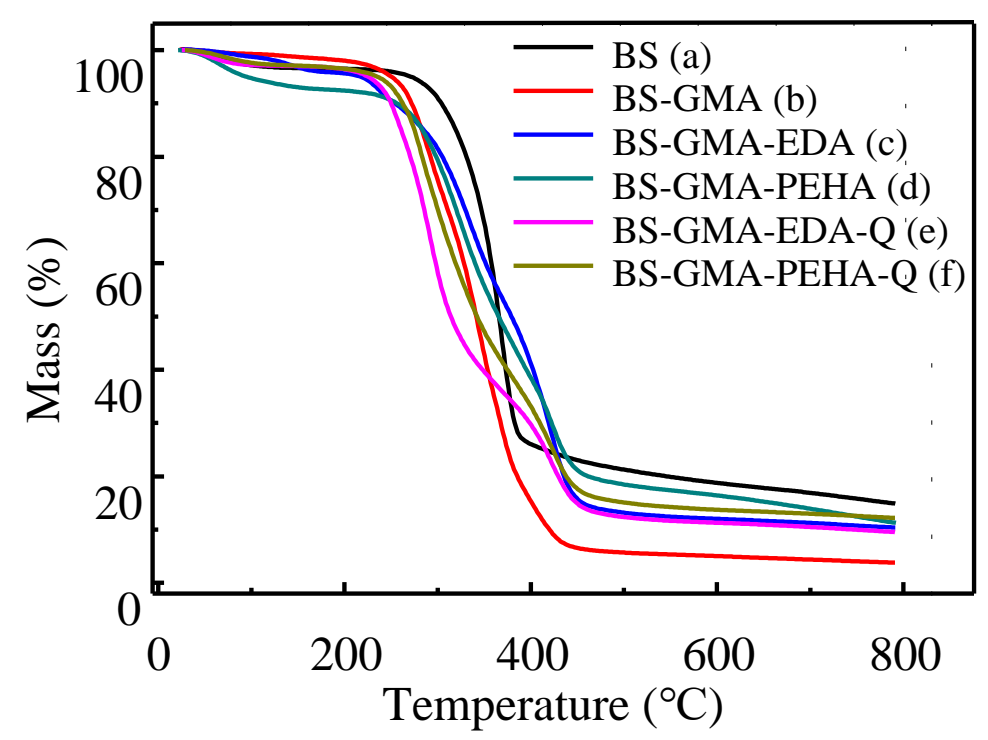

Figure 5. TG analysis for BS (a), BS-GMA (b), BS-GMA-EDA (c), BS-GMA-PHEA (d), BS-GMA-EDA$\mathrm{Q}(\mathrm{e})$, and BS-GMA-PHEA-Q (f).

\subsubsection{XPS Analysis}

XPS analysis was used to provide the element's chemical state on the adsorbent. Figure 6 shows the high-resolution N1s spectra of BS based adsorbents. The N1s spectra of BS-GMA-EDA were curve-fitted into two peaks located at $399.53 \mathrm{eV}$ and $400.55 \mathrm{eV}$. N1s spectra of are BS-GMA-PEHA located at $398.77 \mathrm{eV}$ and $400.33 \mathrm{eV}$ [32]. After quaternization, the peak at 399.53 of BS-GMA-EDA shifted to $402.18 \mathrm{eV}$, and the peak at $398.77 \mathrm{eV}$ shifted to $402.09 \mathrm{eV}$, showing that quaternization was successful [33-35].
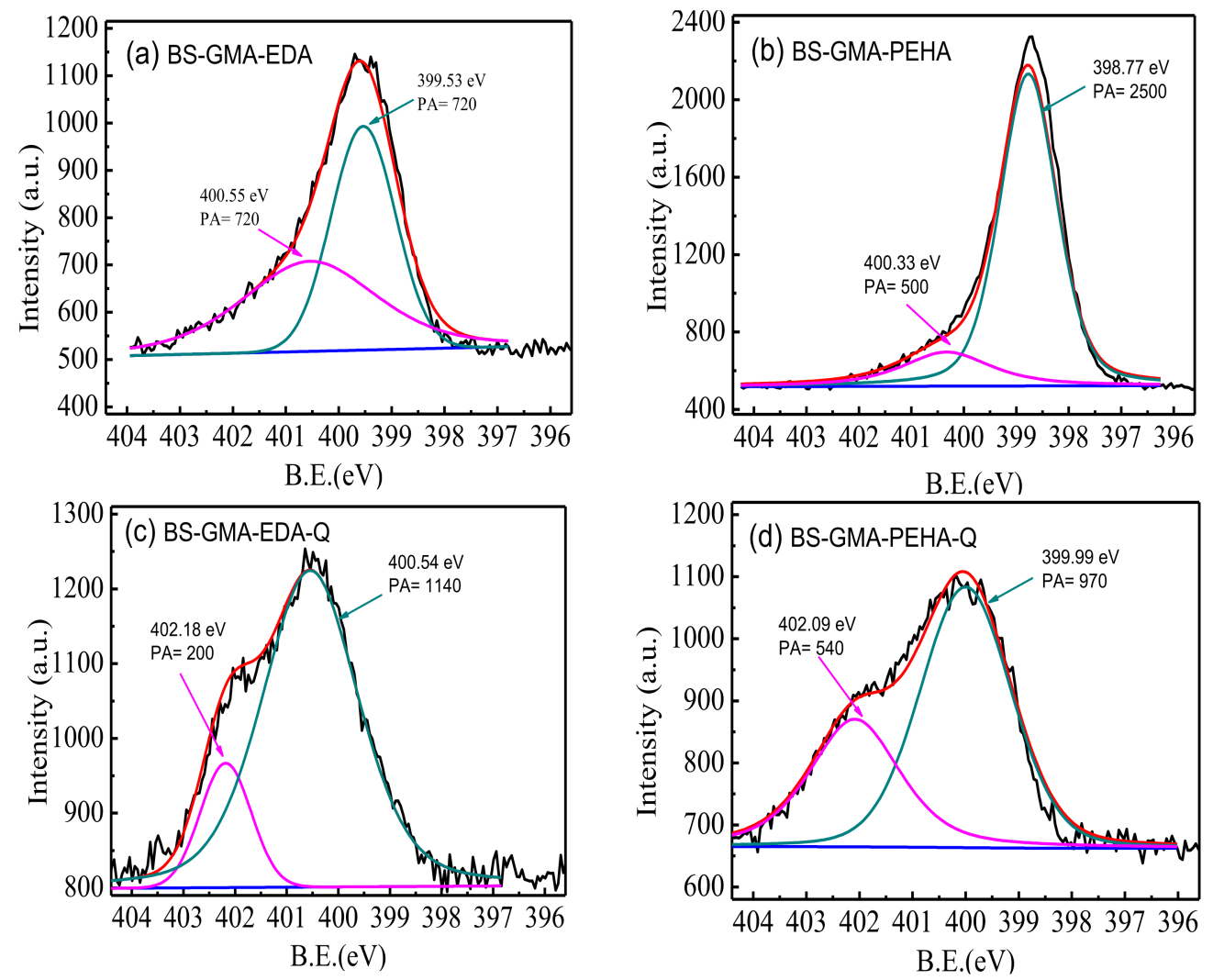

Figure 6. The high-resolution XPS N1s spectra of BS-GMA-EDA (a), BS-GMA-PHEA (b), BS-GMAEDA-Q (c), and BS-GMA-PHEA-Q (d). 


\subsection{Phosphate Adsorption in Batch Experiments}

\subsubsection{Effect of $\mathrm{pH}$}

Figure 7a shows the effect of $\mathrm{pH}$ on phosphate adsorption onto the BS-based adsorbents. Phosphate exists in different ionic species, including $\mathrm{H}_{3} \mathrm{PO}_{4}$, monovalent $\mathrm{H}_{2} \mathrm{PO}^{-}$, divalent $\mathrm{HPO}_{4}{ }^{2-}$, and trivalent $\mathrm{PO}_{4}{ }^{3-}$ ions, depending on the $\mathrm{pH}$ of the solution [36]. At $\mathrm{pHs}$ greater than 2, phosphate mainly exists in the anionic forms of $\mathrm{H}_{2} \mathrm{PO}_{4}{ }^{-}, \mathrm{HPO}_{4}{ }^{2-}$, and $\mathrm{PO}_{4}{ }^{3-}$.
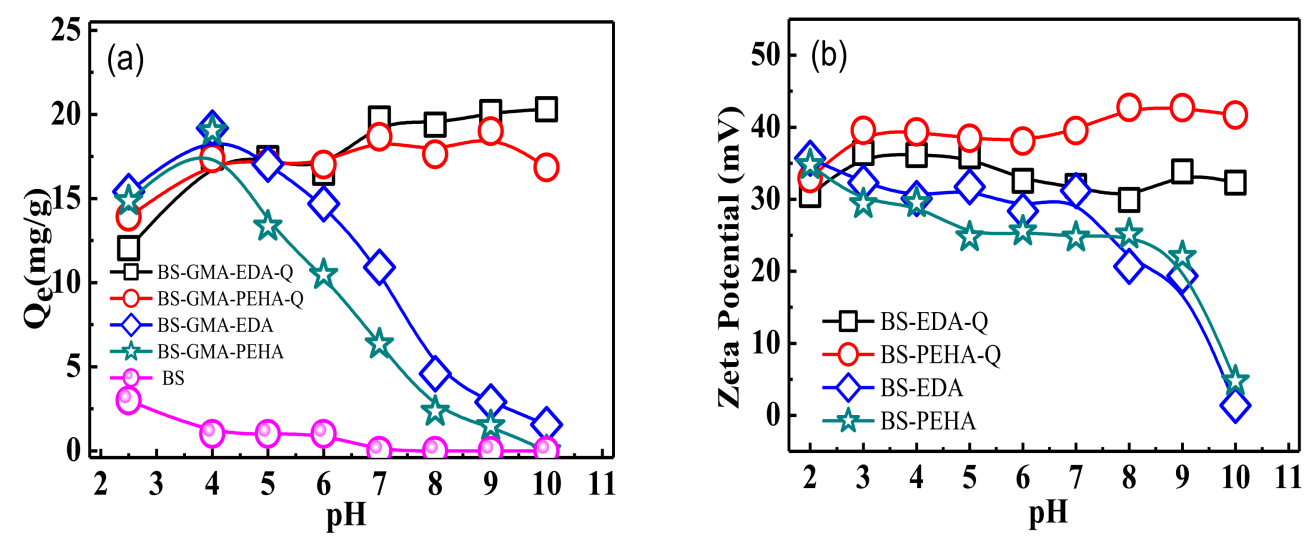

Figure 7. Effect of $\mathrm{pH}$ on phosphate adsorption on the BS based adsorbents (a) and Zeta potential of the adsorbents $(\mathbf{b})$.

BS has a negligible adsorption ability to phosphate at all $\mathrm{pH}$ ranges. Phosphate adsorption by the aminated BS (BS-GMA-EDA and BS-GMA-PEHA) was influenced by varying the $\mathrm{pH}$. The adsorption capacity was higher at acidic conditions than the adsorption capacity at alkaline conditions. At lower $\mathrm{pHs}$, the protonation of the amine makes the adsorbents positively charged, providing electrostatic attraction to the anionic phosphate molecule, thus resulting in a high adsorption performance. At a $\mathrm{pH}$ of 4, BS-GMA-EDA and BS-GMA-PEHA have the highest phosphate adsorption capacity. Below $\mathrm{pH} 4$, the dominant species was transformed to $\mathrm{H}_{2} \mathrm{PO}_{4}{ }^{-}$and neutral $\mathrm{H}_{3} \mathrm{PO}_{4}$, causing the adsorption capacity decrease. At higher $\mathrm{pHs}$, the adsorption capacity decreased due to the de-protonation of the amine and the competition of $\mathrm{OH}^{-}$.

The zeta potential of BS-GMA-EDA and BS-GMA-PEHA explain the $\mathrm{pH}$-dependent adsorption, as shown in Figure $7 \mathrm{~b}$. The zeta potential was higher in acidic conditions than those in alkaline conditions. Thus, the positive value in acidic conditions was favorable for phosphate adsorption. To increase the phosphate adsorption performance of the modified BS in a broader $\mathrm{pH}$ range, BS-GMA-EDA and BS-GMA-PEHA were quaternized.

Unlike BS-GMA-EDA and BS-GMA-PEHA, the phosphate adsorption capacity of BSGMA-EDA-Q and BS-GMA-PEHA-Q was independent of $\mathrm{pH}$. Moreover, the zeta potential was also very high in alkaline conditions. The electrostatic attraction strength between the quaternary ammonium salt group and the phosphate remained unchanged. Thus, the BS-GMA-EDA-Q and BS-GMA-PEHA-Q can absorb phosphate at a broad $\mathrm{pH}$, which is more favorable for a wide range of applications.

\subsubsection{Adsorption Kinetics}

An essential feature of adsorbents is the adsorption kinetics, which allows the measurement of the adsorption rate. Figure 8 a shows the phosphate adsorption capacity of the synthesized adsorbents at different contact times. The phosphate adsorption capacities of BS-GMA-EDA-Q and BS-GMA-PEHA-Q increased rapidly with increasing contact time and reached equilibrium at $180 \mathrm{~min}$. 

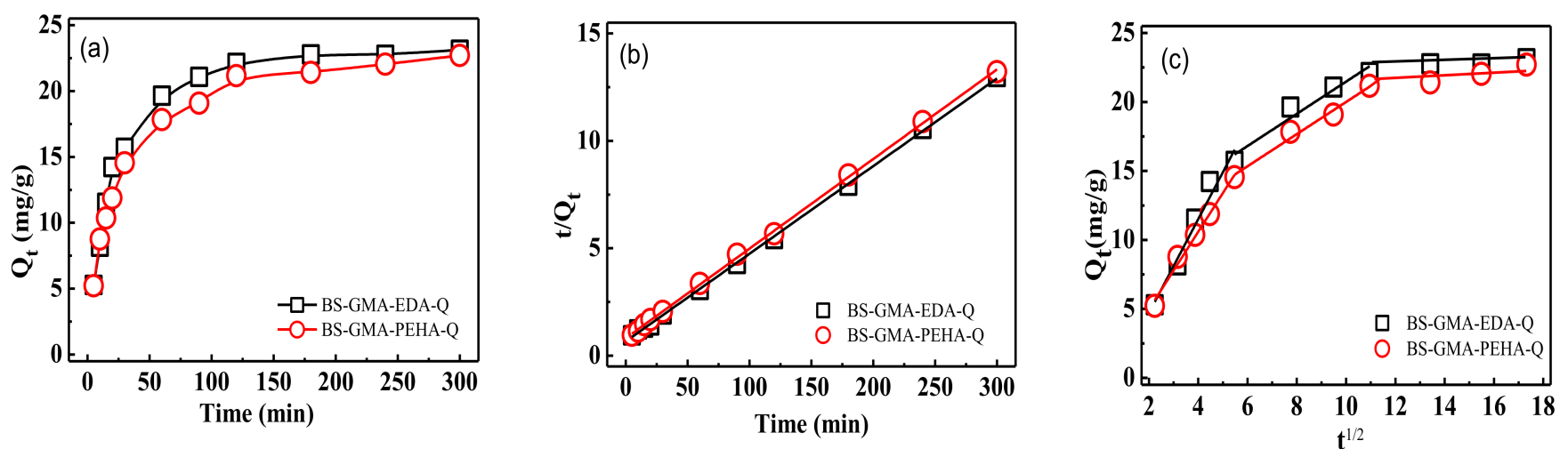

Figure 8. Adsorption kinetics of phosphate removal onto BS in $50 \mathrm{~mL}, 25 \mathrm{mg} / \mathrm{L}, 0.05 \mathrm{~g}$, pH 7, (a) effect of adsorption time; (b) pseudo-second-order; (c) intra-particle diffusion model.

Pseudo-first-order, pseudo-second-order, and Weber-Morris models were used to predict the adsorption mechanism and describe the phosphate adsorption rate of BS-GMAEDA-Q and BS-GMA-PEHA-Q, expressed by Equations (4)-(6), respectively [37].

$$
\begin{gathered}
\ln \left(q_{e}-q_{t}\right)=\ln q_{e}-k_{1} t \\
\frac{t}{q_{t}}=\frac{1}{k_{2} q_{e}^{2}}+\frac{t}{q_{e}} \\
q_{t}=k_{\mathrm{in}} t^{1 / 2}+I
\end{gathered}
$$

where $q_{t}$ and $q_{e}(\mathrm{mg} / \mathrm{g})$ are the amounts of phosphate adsorbed on $1 \mathrm{~g}$ adsorbent at time $\mathrm{t}(\mathrm{min})$ and equilibrium, respectively. $k_{1}$ and $k_{2}$ are the rate constant for the respective model [36]. $K_{\mathrm{in}}$ is the reaction rate constant $\left(\mathrm{mg} / \mathrm{g} \cdot \mathrm{min}^{1 / 2}\right)$, and $I$ is the intercept. The fitting parameters and the correlation coefficients $\left(\mathrm{R}^{2}\right)$ are listed in Table 1.

Table 1. Kinetic parameters obtained from pseudo-first-order, pseudo-second-order kinetic and intra-particle diffusion model.

\begin{tabular}{cccc}
\hline Model & Parameters & BS-GMA-EDA-Q & BS-GMA-PEHA-Q \\
\hline \multirow{2}{*}{ pseudo-first-order } & $k_{1}\left(\mathrm{~h}^{-1}\right)$ & 0.0458 & 0.0421 \\
kinetics & $q_{e}(\mathrm{mg} / \mathrm{g})$ & 22.266 & 21.177 \\
& $\mathrm{R}^{2}$ & 0.9843 & 0.9616 \\
pseudo-second-order & $k_{2}(\mathrm{~g} /(\mathrm{mg} \cdot \mathrm{min}))$ & 0.0025 & 0.0021 \\
kinetics & $q_{e}(\mathrm{mg} / \mathrm{g})$ & 24.504 & 23.981 \\
& $\mathrm{R}^{2}$ & 0.9995 & 0.9995 \\
& $K_{i d 1}$ & 3.401 & 2.8049 \\
Weber-Morris & $I_{1}$ & -2.094 & -0.6285 \\
& $\mathrm{R}^{2}$ & 0.9557 & 0.9860 \\
& $K_{i d 2}$ & 1.1673 & 1.1649 \\
& $I_{2}$ & 9.8019 & 8.3497 \\
$\mathrm{R}^{2}$ & 0.9278 & 0.9775 \\
\hline
\end{tabular}

The correlation coefficients $\left(\mathrm{R}^{2}\right)$ of the pseudo-second-order kinetic model for BSGMA-EDA-Q and BS-GMA-PEHA-Q were 0.9995 and 0.9995, respectively. The high $\mathrm{R}^{2}$ revealed that the pseudo-second-order adsorption mechanism was predominant in the adsorption process. The pseudo-second-order kinetic model shows that there was a chemical adsorption mechanism between the phosphate and the two adsorbents.

The Weber-Morris model was used to investigate whether intra-particle diffusion was the only rate-controlling process in the adsorption process. The Weber-Morris model describe the ions' transport from aqueous solutions to the adsorbent. In this adsorption process, the plots present three distinct linear regions. The initial region represents the 
phosphate transport from the aqueous solution to the BS-GMA-EDA-Q and BS-GMAPEHA-Q surfaces. The second region presents the gradual adsorption corresponding to intra-particle diffusion. The third plateau region indicates equilibrium adsorption. The first linear part did not pass through the origin, indicating that intra-particle diffusion was not the sole rate-determining step [38].

\subsubsection{Adsorption Isotherms}

The adsorption isotherm was used to describe the distribution of the adsorbate molecules on the adsorbents. BS-GMA-EDA-Q and BS-GMA-PEHA-Q adsorption isotherms were conducted at $25^{\circ} \mathrm{C}$ with a phosphate concentration range of $25-250 \mathrm{mg} / \mathrm{L}$.

The correlation between the equilibrium adsorption of $q_{e}(\mathrm{mg} / \mathrm{g})$ and the equilibrium concentration $C_{e}(\mathrm{mg} / \mathrm{L})$ in the solution is shown in Figure 9a. The adsorption capacity of BS-GMA-EDA-Q and BS-GMA-PEHA-Q increased with the increasing phosphate concentration, and finally reached a constant.
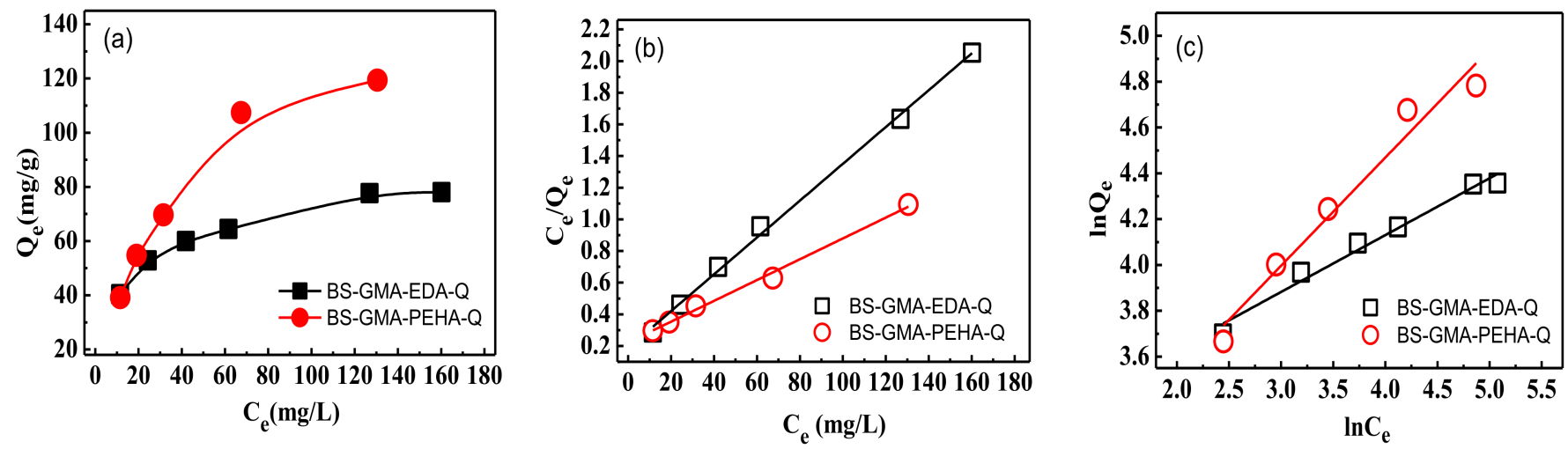

Figure 9. Equilibrium studies of phosphate adsorption: relation of the adsorption capacity with the equilibrium concentration (a), Langmuir (b), and Freundlich model (c).

Langmuir and Freundlich isotherm models were used to fit the adsorption data at various concentrations. The linear equation can be described by Equations (7) and (8) [39].

$$
\begin{aligned}
\frac{C_{e}}{Q_{e}} & =\frac{C_{e}}{Q_{m}}+\frac{1}{K_{L} Q_{m}} \\
\ln Q_{e} & =\ln K_{F}+\frac{1}{n} \ln C_{e}
\end{aligned}
$$

where $C_{e}$ referred the adsorbate concentration $(\mathrm{mg} / \mathrm{L})$ at equilibrium, $Q_{e}$ and $Q_{m}$ were the mass adsorbed on $1 \mathrm{~g}$ adsorbent and the maximum adsorption capacity $(\mathrm{mg} / \mathrm{g})$. Table 2 summarizes the constants of the Langmuir and Freundlich models.

Table 2. Langmuir and Freundlich isotherm parameters and correlation coefficients for the adsorption of phosphate ion.

\begin{tabular}{ccccccc}
\hline \multirow{2}{*}{ Adsorbents } & \multicolumn{3}{c}{ Langmuir } & \multicolumn{3}{c}{ Freundlich } \\
\cline { 2 - 7 } & $\left.\mathbf{Q}_{\mathbf{m}} \mathbf{( m g} / \mathbf{g}\right)$ & $\mathbf{K}_{\mathbf{L}}$ & $\mathbf{R}^{\mathbf{2}}$ & $\left.\mathbf{K}_{\mathbf{F}} \mathbf{( m g} \cdot \mathbf{L}^{-\mathbf{1}}\right)$ & $\mathbf{n}$ & $\mathbf{R}^{\mathbf{2}}$ \\
\hline BS-GMA-EDA-Q & 85.985 & 0.0620 & 0.9972 & 23.055 & 4.0297 & 0.9752 \\
BS-GMA-PEHA-Q & 152.21 & 0.0296 & 0.9931 & 13.209 & 2.1187 & 0.9568 \\
\hline
\end{tabular}

The Langmuir isotherm model assumes that the adsorbate has a monolayer adsorption on the adsorbent through a homogeneous surface. Once an adsorbate molecule was adsorbed on an active adsorption site, no more molecules can be adsorbed on the same site. The maximum adsorption capacity was achieved when all the adsorption sites of 
the adsorbent were saturated with the adsorbate. The Freundlich model was applied to describe adsorption on homogeneous and heterogeneous surfaces. The fitting curves of the Langmuir and Freundlich models are shown in Figure 9b,c, respectively. Comparatively, the Langmuir isotherm model can better describe the adsorption isotherms. The $\mathrm{R}^{2}$ value (0.9972 and 0.9931) of the Langmuir model was very high, suggesting that the adsorbed phosphate forms a monolayer onto the surface of BS-GMA-EDA-Q and BS-GMA-PEHA$\mathrm{Q}$ [40]. The obtained maximum adsorption capacity $\left(\mathrm{Q}_{\max }\right)$ for phosphate by BS-GMAEDA-Q and BS-GMA-PEHA-Q were 85.99 and $152.21 \mathrm{mg} / \mathrm{g}$, respectively. The values were relatively higher compared with other similar adsorbents shown in Table 3.

Table 3. Comparison of adsorption capacity of BS-GMA-EDA-Q and BS-GMA-PEHA-Q with other available sawdust.

\begin{tabular}{cccc}
\hline Adsorbent & $\begin{array}{c}\text { Max Adsorption } \\
\text { Capacity (mg/g) }\end{array}$ & pH & Reference \\
\hline woody sawdust nanoparticles & 50 & 7 & {$[41]$} \\
\hline polypyrrole-coated sawdust & $17.33-30.39$ & & {$[42]$} \\
\hline amine-crosslinked Shaddock Peel & 59.89 & 3 & {$[43]$} \\
\hline diethylamine modified Cellulose & 22.88 & 6.8 & {$[44]$} \\
\hline modified sugar-cane bagasse & 21.3 & 7 & {$[45]$} \\
\hline wheat straw anion exchanger & 52.80 & 6 & {$[46]$} \\
\hline Amine crosslinked tea waste & 98.72 & 7 & This paper \\
\hline BS-GMA-EDA-Q & 85.25 & & {$[40]$} \\
\hline Modified aleppo pine sawdust & 116.25 & 7 & This paper \\
\hline BS-GMA-PEHA-Q & 152.21 & &
\end{tabular}

\subsection{Regeneration}

To evaluate the reusability of BS-GMA-EDA-Q and BS-GMA-PEHA-Q, the adsorptiondesorption cycles were repeated five times using $1 \mathrm{M} \mathrm{HCl}$ as the elution reagent. Figure 10 shows that BS-GMA-EDA-Q and BS-GMA-PEHA-Q have a negligible decrease in adsorption capacity after five adsorption-desorption cycles, indicating that BS-GMA-EDA-Q and BS-GMA-PEHA-Q were stable and recyclable for phosphate removal.

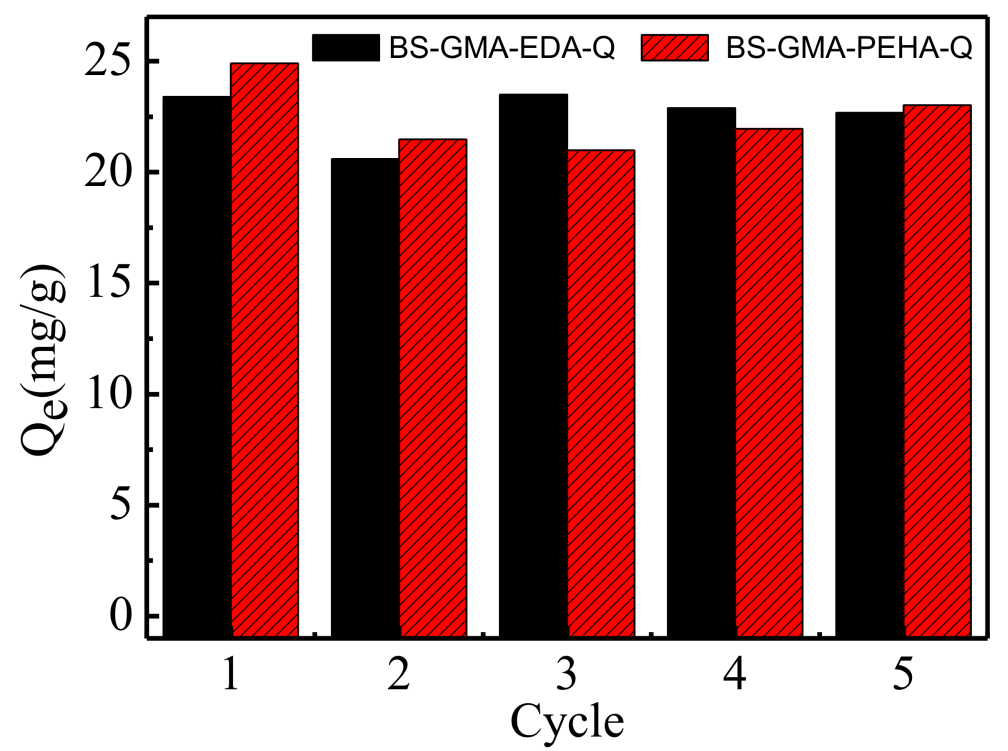

Figure 10. The adsorption-desorption cycles of BS-GMA-EDA-Q and BS-GMA-PEHA-Q for phosphate. 


\section{Conclusions}

Two BS-based adsorbents were successfully synthesized by grafting GMA on BS using the RIGP technique, followed by a ring-opening reaction and quaternization. The adsorption performance of BS-GMA-EDA-Q and BS-GMA-PEHA-Q adsorbents for phosphate removal was investigated.

(1) After quaternization, BS-GMA-EDA-Q and BS-GMA-PEHA-Q showed a high adsorption capacity for phosphate. The adsorption capacity was independent to $\mathrm{pH}$.

(2) The adsorption kinetics of BS-GMA-EDA-Q and BS-GMA-PEHA-Q well obeyed the pseudo-second-order model. The phosphate uptake reached equilibrium within $180 \mathrm{~min}$.

(3) BS-GMA-EDA-Q and BS-GMA-PEHA-Q adsorbents for phosphate removal well obeyed the Langmuir model. The maximum phosphate adsorption capacities of BS-GMA-EDA-Q and BS-GMA-PEHA-Q were 85.25 and $152.21 \mathrm{mg} / \mathrm{g}$, respectively.

(4) The repeated use showed that the adsorption performance of the BS-GMA-EDA-Q and BS-GMA-PEHA-Q adsorbents displayed a negligible decrease after five cycles. Therefore, the BS-GMA-EDA-Q and BS-GMA-PEHA-Q adsorbents exhibit good recyclability and can be used repeatedly in practical applications.

Author Contributions: Conceptualization: Z.D.; formal analysis: J.D., H.X.; investigation: J.D., Z.D.; data curation: X.Y., H.X.; writing—original draft preparation: J.D., J.Y.; writing—review and editing: L.Z., J.Y.; visualization: L.Z.; supervision: L.Z.; funding acquisition: J.D., H.X. All authors have read and agreed to the published version of the manuscript.

Funding: This research was funded by "Hubei Provincial Natural Science Foundation, China, grant number 2020CFB852", Research Project of Department of Education of Hubei Provincial, China, grant number BXLBX0781, and Foundation Project of Hubei University of Science and Technology, grant number BK201820.

Institutional Review Board Statement: Not applicable.

Informed Consent Statement: Not applicable.

Data Availability Statement: The data presented in this study are available on request from the corresponding author.

Conflicts of Interest: The authors declare no conflict of interest.

\section{References}

1. Zhang, L.; Liu, Y.; Li, Z. Effects of reduced severity of ammonium sulfite pretreatment on bamboo for high cellulose recovery. RSC Adv. 2019, 9, 30489-30495. [CrossRef]

2. Xiong, W.; Hu, D. Fabrication of phosphonium bamboo cellulose by triphenylphosphine: Preparation, characterization, and adsorption of Acid Black 24. Environ. Sci. Pollut. Res. 2019, 26, 1880-1891. [CrossRef]

3. Alchouron, J.; Navarathna, C.; Chludil, H.D. Assessing South American Guadua chacoensis bamboo biochar and $\mathrm{Fe}_{3} \mathrm{O}_{4}$ nanoparticle dispersed analogues for aqueous arsenic(V) remediation. Sci. Total Environ. 2020, 706, 135943. [CrossRef] [PubMed]

4. Guan, M.; An, X.; Liu, H. Cellulose nanofiber (CNF) as a versatile filler for the preparation of bamboo pulp based tissue paper handsheets. Cellulose 2019, 26, 2613-2624. [CrossRef]

5. Zhang, Q.; Ning, L.; Shen, Y. Study on shielding effectiveness, electrical conductivity and thermal property of bamboo-plastic shielding composite based on Ni-Fe-P coated bamboo fibers. Mater. Lett. 2020, 268, 127578. [CrossRef]

6. Huang, C.; Tang, S.; Zhang, W.; Tao, Y.; Lai, C.; Li, X.; Yong, Q. Unveiling the structural properties of lignin-carbohydrate complexes in bamboo residues and its functionality as antioxidants and immunostimulants. ACS Sustain. Chem. Eng. 2018, 6, 12522-12531. [CrossRef]

7. Su, Y.; Dong, H.; Li, M. Isolation of the Flavonoid from Bamboo Residues and Its Application as Metal Ion Sensor in vitro. Polymers 2019, 11, 1377. [CrossRef]

8. Chen, H.; Cheng, Y.; Zhu, Z.; He, H.; Zhu, Y. Adsorption of Pb(II) from Aqueous Solution by Mercerized Moso Bamboo Chemically Modified with Pyromellitic Dianhydride. J. Environ. Eng. 2020, 146, 04019127. [CrossRef]

9. Zhao, X.; Zeng, T.; Li, X.; Hu, Z.; Gao, H.; Xie, Z. Modeling and mechanism of the adsorption of copper ion onto natural bamboo sawdust. Carbohydr. Polym. 2012, 89, 185-192. [CrossRef]

10. Hata, M.; Amano, Y.; Thiravetyan, P.; Machida, M. Preparation of Bamboo Chars and Bamboo Activated Carbons to Remove Color and COD from Ink Wastewater. Water Environ. Res. 2016, 88, 87-96. [CrossRef] 
11. Jawad, A.H.; Abdulhameed, A.S. Statistical modeling of methylene blue dye adsorption by high surface area mesoporous activated carbon from bamboo chip using $\mathrm{KOH}$-assisted thermal activation. Energy Ecol. Environ. 2020, 5, 456-469. [CrossRef]

12. Reddy, P.; Krushnamurty, K.; Mahammadunnisa, S.K. Preparation of activated carbons from bio-waste: Effect of surface functional groups on methylene blue adsorption. Int. J. Environ. Sci. Technol. 2015, 12, 1363-1372. [CrossRef]

13. Zhang, J.; Shang, T.; Jin, X.; Gao, J.; Zhao, Q. Study of chromium(VI) removal from aqueous solution using nitrogen-enriched activated carbon based bamboo processing residues. RSC Adv. 2015, 5, 784-790. [CrossRef]

14. Hou, Y.; Yan, S.; Huang, G.; Yang, Q.; Huang, S.; Cai, J. Fabrication of N-doped carbons from waste bamboo shoot shell with high removal efficiency of organic dyes from water. Bioresour. Technol. 2020, 303, 122939. [CrossRef] [PubMed]

15. Peng, X.; Hu, F.; Zhang, T.; Qiu, F.; Dai, H. Amine-functionalized magnetic bamboo-based activated carbon adsorptive removal of ciprofloxacin and norfloxacin: A batch and fixed-bed column study. Bioresour. Technol. 2017, 249, 924-934. [CrossRef]

16. Zhou, S.K.; Liu, Y.J.; Jiang, H.Y.; Deng, W.J.; Zeng, G.M. Adsorption of U(VI) from Aqueous Solution by a Novel Chelating Adsorbent Functionalized with Amine Groups: Equilibrium, Kinetic, and Thermodynamic Studies. Environ. Eng. Sci. 2017, 35, 1-9. [CrossRef]

17. Wang, S.; Wang, L.; Kong, W.; Ren, J.; Liu, C.; Wang, K.; Sun, R.; She, D. Preparation, characterization of carboxylated bamboo fibers and their adsorption for lead(II) ions in aqueous solution. Cellulose 2013, 20, 2091-2100. [CrossRef]

18. Zhang, X.; Zhao, J.; Cheng, L.; Lu, C.; Wang, Y.; He, X.; Zhang, W. Acrylic acid grafted and acrylic acid/sodium humate grafted bamboo cellulose nanofibers for $\mathrm{Cu}^{2+}$ adsorption. RSC Adv. 2014, 4, 55195-55201. [CrossRef]

19. Hou, C.; Wu, Y.; Wang, T.; Gao, X. Preparation of Quaternized Bamboo Cellulose and Its Implication in Direct Air Capture of $\mathrm{CO}_{2}$. Energy Fuel. 2019, 33, 1745-1752. [CrossRef]

20. Zhao, J.; Wang, L.; Xiao, J.; Tao, M.; Zhang, W. Removal of anionic azo dyes from aqueous solutions by quaternary ammonium salt-functionalized fibers with adjustable surface micro environments. React. Funct. Polym. 2020, 154, 104684. [CrossRef]

21. Du, J.F.; Dong, Z.; Pi, Y.; Yang, X.; Zhao, L. Fabrication of Cotton Linter-Based Adsorbents by Radiation Grafting Polymerization for Humic Acid Removal from Aqueous Solution. Polymers 2019, 11, 962. [CrossRef]

22. Yu, X.; Tong, S.; Ge, M.; Wu, L.; Song, W. Synthesis and characterization of multi-amino-functionalized cellulose for arsenic adsorption. Carbohyd. Polym. 2013, 92, 380-387. [CrossRef]

23. Xu, C.; Yu, T.; Peng, J.; Zhao, L.; Li, J.; Zhai, M. Efficient Adsorption Performance of Lithium Ion onto Cellulose Microspheres with Sulfonic Acid Groups. Quantum Beam Sci. 2020, 4, 6. [CrossRef]

24. Du, J.F.; Dong, Z.; Yang, X.; Zhao, L. Facile fabrication of sodium styrene sulfonate-grafted ethylene- vinyl alcohol copolymer as adsorbent for ammonium removal from aqueous solution. Environ. Sci. Pollut. Res. 2018, 25, 27235-27244. [CrossRef] [PubMed]

25. Hao, H.; Wang, Y.; Shi, B. NaLa $\left(\mathrm{CO}_{3}\right)_{2}$ hybridized with $\mathrm{Fe}_{3} \mathrm{O}_{4}$ for efficient phosphate removal: Synthesis and adsorption mechanistic study. Water Res. 2019, 155, 1-11. [CrossRef]

26. Wang, B.; Zhang, W.; Li, L.; Guo, W.; Hong, Z. Novel talc encapsulated lanthanum alginate hydrogel for efficient phosphate adsorption and fixation. Chemosphere 2020, 256, 127124. [CrossRef] [PubMed]

27. Asaoka, S.; Kawakami, K.; Saito, H. Adsorption of phosphate onto lanthanum-doped coal fly ash-Blast furnace cement composite. J. Hazard. Mater. 2020, 406, 124780. [CrossRef] [PubMed]

28. Wang, Y.C.; Peng, J.; Li, J.Q.; Zhai, M.L. PVDF based ion exchange membrane prepared by radiation grafting of ethyl styrenesulfonate and sequent hydrolysis. Radiat. Phys. Chem. 2017, 130, 252-258. [CrossRef]

29. Chattopadhyay, D.; Umrigar, K. Chemical Modification of Waste Cotton Linters for Oil Spill Cleanup Application. J. Inst. Eng. India Ser. E 2017, 98, 103-120. [CrossRef]

30. Lv, X.; Li, Y.; Yang, M. Humidity sensitive properties of copolymer of quaternary ammonium salt with polyether-salt complex. Polym. Adv. Technol. 2009, 20, 509-513. [CrossRef]

31. Deng, S.; Zheng, Y.; Xu, F.; Wang, B.; Huang, J.; Yu, G. Highly efficient sorption of perfluorooctane sulfonate and perfluorooctanoate on a quaternized cotton prepared by atom transfer radical polymerization. Chem. Eng. J. 2012, 193, 154-160. [CrossRef]

32. Liang, R.H.; Li, Y.; Huang, L.; Wang, X.D.; Hu, X.; Liu, C.M.; Chen, M.S.; Chen, J. Pb ${ }^{2+}$ adsorption by ethylenediamine-modified pectins and their adsorption mechanisms. Carbohyd. Polym. 2020, 234, 115911. [CrossRef]

33. Hu, G.W.; Wang, Y.; Ma, J.; Qiu, J.Y.; Peng, J.; Li, J.Q.; Zhai, M.L. A novel amphoteric ion exchange membrane synthesized by radiation-induced grafting $\alpha$-methylstyrene and $\mathrm{N}, \mathrm{N}$-dimethylaminoethyl methacrylate for vanadium redox flow battery application. J. Membr. Sci 2012, 407-408, 184-192. [CrossRef]

34. Cao, W.; Wang, Z.; Zeng, Q.; Shen, C. ${ }^{13} \mathrm{C}$ NMR and XPS characterization of anion adsorbent with quaternary ammonium groups prepared from rice straw, corn stalk and sugarcane bagasse. Appl. Surf. Sci. 2016, 389, 404-410. [CrossRef]

35. Kavakli, C.; Kavakli, P.A.; Turan, B.D.; Hamurcu, A.; Guven, O. Quaternized dimethylaminoethyl methacrylate strong base anion exchange fibers for As(V) adsorption. Radiat. Phys. Chem. 2014, 102, 84-95. [CrossRef]

36. Du, J.F.; Dong, Z.; Lin, Z.; Yang, X.; Zhao, L. Radiation Synthesis of Pentaethylene Hexamine Functionalized Cotton Linter for Effective Removal of Phosphate: Batch and Dynamic Flow Mode Studies. Materials 2019, 12, 3393. [CrossRef] [PubMed]

37. Tejada-Tovar, C.; Villaboni-Ortiz, A.; Ortega-Toro, R.; Mancilla-Bonilla, H.; Espinoza-Leon, F. Potential Use of Residual Sawdust of Eucalyptus globulus Labill in $\mathrm{Pb}$ (II) Adsorption: Modelling of the Kinetics and Equilibrium. Appl. Sci. 2021, 11, 3125. [CrossRef]

38. Ma, Z.; Li, Q.; Yue, Q.; Gao, B.; Li, W.; Xu, X.; Zhong, Q. Adsorption removal of ammonium and phosphate from water by fertilizer controlled release agent prepared from wheat straw. Chem. Eng. J. 2011, 171, 1209-1217. [CrossRef] 
39. Alorabi, A.Q.; Alharthi, F.A.; Azizi, M.; Al-Zaqri, N.; Elmarghany, A.; Abdelshafeek, K. Removal of Lead(II) from Synthetic Wastewater by Lavandula Pubescens Decne Biosorbent: Insight into Composition-Adsorption Relationship. Appl. Sci. 2020, 10, 7450. [CrossRef]

40. Qiao, H.; Mei, L.; Chen, G.; Liu, H.; Peng, C.; Ke, F.; Hou, R.; Wan, X.; Cai, H. Adsorption of nitrate and phosphate from aqueous solution using amine cross-linked tea wastes. Appl. Surf. Sci. 2019, 483, 114-122. [CrossRef]

41. Mahdy, A.M.; Salem, M.Z.M.; Ali, A.M.; Ali, H.M. Optimum Operating Conditions for the Removal of Phosphate from Water Using of Wood-Branch Nanoparticles from Eucalyptus camaldulensis. Materials 2020, 13, 1851. [CrossRef]

42. Bajpai, S.K.; Rohit, V.K.; Namdeo, M. Removal of phosphate anions from aqueous solutions using polypyrrole-coated sawdust as a novel sorbent. J. App. Polym. Sci. 2010, 111, 3081-3088. [CrossRef]

43. Duan, P.; Xu, X.; Shang, Y.; Gao, B.; Li, F. Amine-crosslinked Shaddock Peel embedded with hydrous zirconium oxide nanoparticles for selective phosphate removal in competitive condition. J. Taiwan Inst. Chem. Eng. 2017, 80, 650-662. [CrossRef]

44. Fan, C.H.; Zhang, Y.C. Adsorption isotherms, kinetics and thermodynamics of nitrate and phosphate in binary systems on a novel adsorbent derived from corn stalks. J. Geochem. Explor. 2018, 188, 95-100. [CrossRef]

45. Zhang, J.; Shan, W.; Ge, J.; Shen, Z.; Lei, L.; Wang, W. Kinetic and Equilibrium Studies of Liquid-Phase Adsorption of Phosphate on Modified Sugarcane Bagasse. J. Environ. Eng. 2012, 138, 252-258. [CrossRef]

46. Xu, X.; Gao, B.Y.; Yue, Q.Y.; Zhong, Q.Q. Preparation of agricultural by-product based anion exchanger and its utilization for nitrate and phosphate removal. Bioresour. Technol. 2010, 101, 8558-8564. [CrossRef] [PubMed]

47. Benyoucef, S.; Amrani, M. Removal of Phosphate from Aqueous Solution with Modified Sawdust. Procedia Eng. 2012, 33, 58-69. [CrossRef] 